\title{
Fermi acceleration at relativistic shocks and its radiative signatures
}

\author{
Brian Reville \\ Clarendon Laboratory, University of Oxford, Parks Road, Oxford OX1 3PU, United Kingdom \\ E-mail: b.reville1@physics.ox.ac.uk
}

John G. Kirk

Max-Planck-Institut für Kernphysik, Saupfercheckweg 1, D-69117 Heidelberg, Germany

E-mail: john.kirk@mpi-hd.mpg.de

\begin{abstract}
The first-order Fermi process at relativistic shocks requires the generation of strong turbulence in the vicinity of the shock front. Recent particle in cell simulations have demonstrated that this mechanism can be studied self-consistently at weakly magnetised shocks. The radiative signature of this first-order Fermi acceleration mechanism is important for models of both the prompt and afterglow emission in gamma-ray bursts. Building on the insight provided by particle in cell simulations, we present possible radiative signatures and show how these can be used as a probe of the local plasma conditions in the source.
\end{abstract}

25th Texas Symposium on Relativistic Astrophysics - TEXAS 2010

December 06-10, 2010

Heidelberg, Germany 


\section{Introduction}

Particle acceleration at relativistic shocks is thought to proceed via the first order Fermi acceleration mechanism (see for example [5]). Recent particle in cell (PIC) simulations have demonstrated that the process can indeed occur, $[13,9,14,15]$ although to date only for the case of unmagnetised or subluminal shocks. These simulations represent a significant advance in the study of Fermi acceleration at relativistic shocks. Most importantly, they are ab initio, in the sense that the process is reproduced from Maxwell's equations and the equations of motion.

While the maximum energy to which a particle is accelerated can be determined by several factors, such as the shock's lifetime or its spatial extent, the acceleration will ultimately cease when the radiative energy losses that are inevitably associated with the scattering process overwhelm the energy gains obtained upon crossing the shock.

Such effects are usually not included in PIC simulations because of the difficulties associated with radiation reaction. However, using the small angle scattering approximation, it is possible to derive an approximate upper limit for the maximum particle energy, and similarly the maximum photon energy. The exact values of these quantities will depend on the details of the scattering process.

\section{Particle transport and acceleration}

For the purpose of estimating radiative signatures of accelerated particles, it is convenient to characterise the fluctuations in terms of their 'strength' or 'wiggler' parameter $a$, defined as the ratio of their length scale $\lambda$ to the length defined by the magnitude of the typical fluctuations in the field strength: $a=\lambda e|\delta B| / m c^{2}$ [7]. Using this strength parameter, the transport can be divided into two distinct regimes, which we call 'ballistic' $(a<\gamma)$ and 'helical' $(a>\gamma)$. In ballistic transport, the scattering mean free path is shorter than the gyroradius in the local field, and the particles move rectilinearly between collisions with the magnetic fluctuation. On the other hand, in the helical transport regime, particles gyrate about their local mean field while their pitch angles and guidingcentre positions diffuse. In the case of oblique shocks, cross-field diffusion must operate at or near the Bohm limit [1]. While the diffusion coefficient is different in the two regimes, it is possible in both cases to derive an upper limit to the particle Lorentz factor when radiative cooling is included.

\section{Ballistic transport regime:}

At a relativistic shock, with Lorentz factor $\bar{\gamma}$, a particle that crosses from downstream to upstream remains in the upstream medium until it has been deflected, on average, through an angle of $1 / \bar{\gamma}$ in the upstream rest frame. After returning downstream the particle must deflect through a much larger angle $\sim \pi / 2$ before it can cross back into the upstream. A turbulent fluctuation of strength parameter $a$, deflects a particle of Lorentz factor $\gamma$ through an angle $a / \gamma$. Provided this angle is small, the diffusion coefficient is simply $\mathscr{D}_{\theta}=a^{2} v_{\mathrm{sc}} / \gamma^{2}$, where $v_{\mathrm{sc}}$ is the mean scattering frequency. The average number of scatterings in the upstream medium between shock encounters is therefore $N_{\text {scatt,u }} \approx\left(\gamma / a_{\mathrm{u}} \bar{\gamma}\right)^{2}$, and likewise in the downstream $N_{\text {scatt,d }} \approx\left(\gamma / a_{\mathrm{d}}\right)^{2}$. At each scattering, the minimum power radiated in photons by the energetic particle can be estimated from Larmor's 
formula:

$$
\left.\frac{\Delta \gamma}{\gamma}\right|_{\text {loss }} \approx \frac{2 a^{2} e^{2} \gamma}{3 m c^{2} \lambda}
$$

Assuming similar strength parameters either side of the shock, radiative losses in the downstream dominate. For kinematic reasons, the average energy gain per cycle is roughly a factor of two [1], so that the acceleration process will saturate when the energy lost in the downstream medium is roughly $\gamma m c^{2}$. This implies that the energy is limited to [6]

$$
\gamma<\left(\frac{3 m c^{2} \lambda_{\mathrm{d}}}{2 e^{2}}\right)^{1 / 3}
$$

The inclusion of other loss processes, such as inverse-Compton cooling, or escape from the shock front, can reduce this limit further still.

\section{Helical transport regime:}

In the helical transport regime, energy losses are important at all points along a trajectory. In the Bohm limit, the maximum Lorentz factor is [1]:

$$
\gamma<\left(\frac{3 m^{2} c^{3}}{2 e^{3} B}\right)^{1 / 2}
$$

At magnetised, relativistic shocks, particle acceleration by the first-order Fermi mechanism is less plausible, since it relies on strong cross-field diffusion. However, if the process does operate, a particle can move from the helical to the ballistic regime, as its Lorentz factor increases. There exists a critical strength parameter $a_{\text {crit }}$ such that when $a=a_{\text {crit }}$ the maximum Lorentz factor $\gamma_{\max }$ is achieved just at the point at which the transport changes character from helical to ballistic, i.e.

$$
a_{\text {crit }}=\left(\frac{3 m c^{2} \lambda_{\mathrm{d}}}{2 e^{2}}\right)^{1 / 3}
$$

If $a>a_{\text {crit }}$, all particles remain in the the helical regime. On the other hand, if $a<a_{\text {crit }}$, particles of the maximum Lorentz factor undergo ballistic transport, but lower energy particles may be in the helical regime.

Combining the constraints from the ballistic regime (2.2) and the helical regime (2.3) gives:

$$
\gamma_{\text {max }}= \begin{cases}a_{\text {crit }} & \text { for } a<a_{\text {crit }} \\ a_{\text {crit }} \sqrt{a_{\text {crit }} / a} & \text { for } a>a_{\text {crit }}\end{cases}
$$

\section{Radiative signatures}

The maximum energy photons produced by the highest energy particles can also be understood in terms of the strength parameter $a$. The character of the emission depends crucially on the "formation" or "coherence" length of the radiated photons. This is determined from the time 


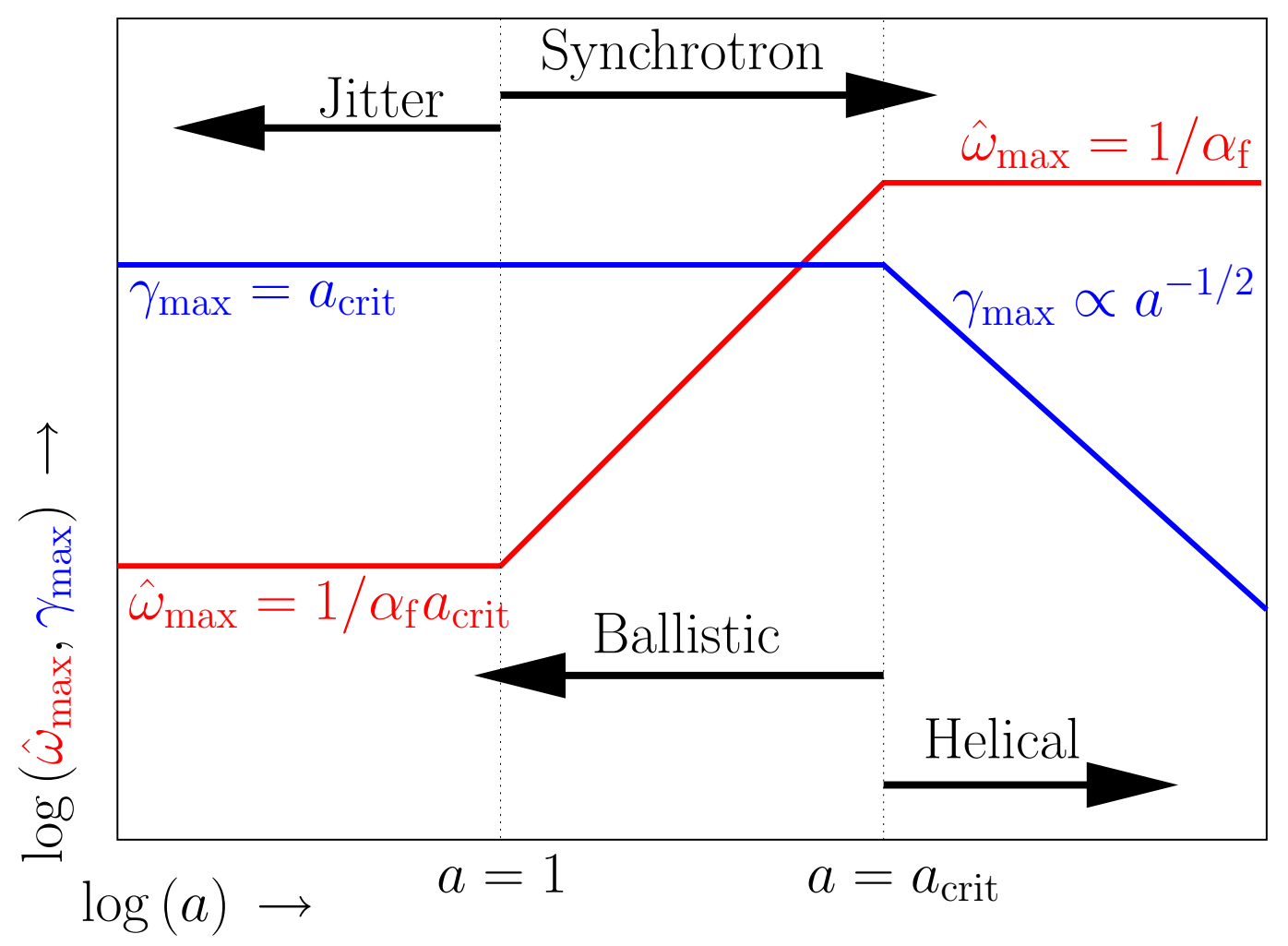

Figure 1: The maximum electron Lorentz factor $\gamma_{\max }$ and the maximum photon energy $\hat{\omega}_{\max }=\hbar \omega_{\max } / m c^{2}$ radiated when scattered by magnetic fluctuations of strength $a$ at a relativistic shock. The jitter/synchrotron regimes are separated by the vertical $a=1$ line; the ballistic/helical transport regimes by the $a=a_{\text {crit }}$ line.

taken for a photon to travel a distance of at least one wavelength ahead of the particle. If $a>1$, the particle sweeps through an angle greater than $1 / \gamma$ during a scattering event. The formation length is $l_{\text {coh }} \approx m c^{2} / e B$, which is smaller than the wavelength of the turbulence, so that the individual photons are created in regions in which the field is almost constant and homogeneous. In this case the radiation is given by the standard synchrotron analysis, with the emissivity defined by the local value of the field. The emission extends up to the roll-over frequency of the highest energy electrons:

$$
\omega_{\max } \approx 0.5 a \gamma_{\max }^{2} c / \lambda \quad \text { for } a>1
$$

If, on the other hand, $a<1$, the particle is deflected through an angle that is small compared to $1 / \gamma$ in a single scattering event. In this case, the coherence length is no longer limited by deflection, but is given by the distance moved by the particle in the lab. frame during the time it takes for the photon to move one wavelength ahead of the particle: $l_{\text {coh }} \approx \gamma^{2} c / \omega$. The maximum frequency is given by the Doppler boosted crossing time of the fluctuation [7]

$$
\omega_{\max } \approx 0.5 \gamma_{\max }^{2} c / \lambda \quad \text { for } a<1
$$


In each case, most of the power radiated by an individual electron emerges within a decade of the roll-over frequency that corresponds to its Lorentz factor. Therefore, the spectrum radiated by a power-law distribution of electrons, with differential number density $\mathrm{d} n / \mathrm{d} \gamma \propto \gamma^{-p}$, for both the synchrotron and jitter cases, reproduces the standard power-law spectrum at frequencies between the roll-over frequency of the highest and lowest energy electrons: $\mathrm{d} L / \mathrm{d} \omega \propto \omega^{-(p-1) / 2}$.

Combining the limit on the Lorentz factor (2.2) with the expressions for the roll-over frequency (3.1) and (3.2), one finds for the maximum frequency that can be radiated by particles accelerated at a relativistic shock front:

$$
\frac{\hbar \omega_{\max }}{m c^{2}}= \begin{cases}\left(\alpha_{\mathrm{f}} a_{\text {crit }}\right)^{-1} & a<1 \\ a\left(\alpha_{\mathrm{f}} a_{\text {crit }}\right)^{-1} & 1<a<a_{\text {crit }} \\ \alpha_{\mathrm{f}}^{-1} & a>a_{\text {crit }}\end{cases}
$$

where $\alpha_{\mathrm{f}}=e^{2} / \hbar c$ is the fine structure constant. The results are summarised in Fig. 1.

\section{Relativistic shock parameters}

So far, the discussion of the acceleration and resulting radiation has been completely general. To relate to observations, it is necessary to make estimates of the relevant parameters.

Relativistic shocks are typically defined in terms of their magnetisation parameter. For a pair plasma

$$
\sigma=B^{2} /\left(8 \pi \bar{\gamma} n m c^{2}\right)
$$

where $n$ is the number density of electrons or positrons. PIC simulations show fundamental differences between the magnetised and unmagnetised cases, the separation between the two regimes occurring at approximately $\sigma \sim 10^{-3}$.

For sufficiently weakly magnetised shocks, or shocks that are almost purely parallel, the field is generated via the Weibel instability. These typically have magnetic structures of size on the order of the plasma skin-depth $\lambda=\ell_{\mathrm{w}} c / \omega_{\mathrm{p}}$ where $\omega_{\mathrm{p}}$ is the local plasma frequency, and $\ell_{\mathrm{w}} \sim 10$.

Magnetised shocks, on the other hand, appear to be mediated by the synchrotron maser instability. In this case, the characteristic length-scale in the downstream plasma $\lambda_{\mathrm{s}, \mathrm{d}}$ is dictated by the requirement that the incoming particles be significantly deflected, giving $\lambda_{\mathrm{s}}=\ell_{\mathrm{s}} \bar{\gamma} m c^{2} / e B_{\mathrm{d}}$ with $\ell_{\mathrm{s}} \sim 1[8]$.

It follows that for a given shock, the critical strength parameter is

$$
a_{\text {crit }} \approx\left\{\begin{array}{l}
10^{6} \ell_{\mathrm{w}}^{1 / 3} \bar{\gamma}^{1 / 6}\left(n / 1 \mathrm{~cm}^{3}\right)^{-1 / 6} \text { Weib } \\
10^{5} \ell_{\mathrm{s}}^{1 / 3} \bar{\gamma}^{1 / 3}(B / 1 \mu \mathrm{G})^{-1 / 3} \text { Synch }
\end{array}\right.
$$

While these results are derived assuming a electron-positron plasma, the dependence on mass is weak, and for an electron-ion shock, $a_{\text {crit }}$ will increase by less than an order of magnitude.

While current PIC simulations suggest strength parameters $\sim \bar{\gamma}$ [14], this result is most likely dependent on the mass ratio of the background species. In addition, only a narrow range of shock Lorentz factors have been investigated, and how this result extends up to the large Lorentz factors expected, for example, at the termination shocks of pulsar winds, is uncertain. 


\section{Conclusions}

Radiation emitted by relativistic electrons scattering in the small-scale turbulent magnetic fields generated at Weibel-mediated relativistic shocks has been proposed as the mechanism responsible for both the prompt and afterglow emission of gamma-ray bursts. The evidence in favour of this suggestion is based on modelling the observed spectra assuming an electron distribution of power-law type with arbitrary high and low energy cut-offs. Power-law distributions are expected on theoretical grounds, and are indeed observed in simulations of weakly magnetized, relativistic shocks $[13,9,14,15]$. However, the constraint on the maximum photon energy imposed by the above analysis (3.3) suggests that this picture is not self-consistent, because the scatterings are too weak to accelerate electrons to the required Lorentz factor. In order to radiate photons of energy $\sim m c^{2}$ in the plasma rest frame, strong fluctuations of large length-scale with $a \sim \alpha_{\mathrm{f}} a_{\text {crit }} \sim 10^{4}$ are required.

The above conclusion rests on the assumption that the same fluctuations are responsible for both the particle transport and radiation. In terms of the strength parameter $a$ and length scale $\lambda$ that we use to characterize the fluctuations, the deflection angle scales as $\Delta \theta \propto a$ and the radiation losses as $\Delta \gamma \propto a^{2} / \lambda$. If, therefore, the scattering responsible for isotropisation occurs on fluctuations of comparable strength, but much larger length scale than those responsible for the radiation losses, the limit on the maximum photon energy (3.3) is relaxed. In principle, the fluctuations induced by the Weibel instability could be responsible for photon production, provided longer wavelength fluctuations are present to provide the necessary isotropisation and transport. The accelerated particles themselves appear to generate longer wavelength fluctuations downstream of the shock [4], but this is a relatively small effect compared to that needed to significantly influence the maximum photon energy.

On the other hand, if, as simulations suggest, the Weibel-induced fluctuations are responsible for the transport, the bulk of the radiation must be produced by interaction with fluctuations of much shorter wavelength. An obvious candidate is the soft photon field produced by the interaction of thermal electrons with the Weibel-induced fluctuations - the 'jitter' analogue of the synchrotron photons produced by relativistic thermal electrons [10, 3, 12]. With these photons as targets, the radiation mechanism is analogous to the synchrotron self-Compton mechanism, which has been discussed in connection with the problem of rapidly decaying magnetic fluctuations [11,2].

\section{Acknowledgments}

BR was supported by an Alexander von Humboldt fellowship for this research

\section{References}

[1] Achterberg, A., Gallant, Y. A., Kirk, J. G., \& Guthmann, A. W. (2001), MNRAS, 328, 393

[2] Baring, M. G., \& Braby, M. L. 2004, ApJ, 613, 460

[3] Fleishman, G. D. 2006, ApJ, 638, 348

[4] Keshet, U., Katz, B., Spitkovsky, A., \& Waxman, E. 2009, ApJ, 693, L127 
[5] Kirk, J. G., \& Duffy, P. 1999, Journal of Physics G Nuclear Physics, 25, 163

[6] Kirk, J. G., \& Reville, B., 2010, ApJL, 710, L16

[7] Landau, L. D., \& Lifshitz, E. M. 1975, The classical theory of fields (Oxford: Pergamon Press, 1975)

[8] Lyubarsky, Y. 2006, ApJ, 652, 1297

[9] Martins, S. F., Fonseca, R. A., Silva, L. O., \& Mori, W. B. 2009, ApJ, 695, L189

[10] Medvedev, M. V. 2000, ApJ, 540, 704

[11] Rossi, E., \& Rees, M. J. 2003, MNRAS, 339, 88

[12] Reville, B. \& Kirk, J. G. 2010, ApJ, 724, 1283

[13] Spitkovsky, A. 2008, ApJL, 682, L5

[14] Sironi, L., \& Spitkovsky, A. 2009, ApJ, 698, 1523

[15] Sironi, L., \& Spitkovsky, A. 2011, ApJ, 726, 75 